\title{
EFFECTS OF ADHESIVE AND LOADING DIRECTIONS ON THE LOAD-CARRYING CAPACITY OF V-NAILS
}

\author{
Hamid R. Taghiyari ${ }^{1, »}$, Mohammad Ghofrani ${ }^{1}$, Farzad Arbabi Ghamsari ${ }^{1}$
}

\begin{abstract}
Effects of tensile and compression loading on the overall strength of two miter frame corner joints, namely V-nail and dovetail joints, with and without adhesive were studied for medium-density fiberboard and particleboard. Two sizes of V-nails were chosen, sizes 7 and 10 . Tensile and compression load-carrying capacity values were measured on the internal and external corners of the joints. Results showed that both wood-composites had greater load-carrying capacities for compression stress in comparison to tensile stress. Medium-density fiberboard demonstrated higher strength compared to particleboard specimens because of its higher compactness of fibers and more integrity in the composite matrix. Application of adhesive resulted in a significant increase in the strength of all joints and both composites. The increase in medium-density fiberboard was significantly higher in comparison to particleboard because lower permeability in medium-density fiberboard did not let adhesive to be uselessly penetrated into the texture. In particleboard specimens, however, adhesive penetrated into the voids and spaces in the composite, and the adhesive line was weakened between the surfaces of the composite bodies. It was concluded that V-nails are recommended for craftsmen in case adhesive is used. However, dovetail joints provide higher strength if the production process necessitates not to use adhesive in joints.
\end{abstract}

Keywords: Cabinet-making industry, loading direction, medium-density fiberboard, particleboard stress, wood-composite panels.

\section{INTRODUCTION}

Many types of joints have so far been presented to cabinet-making industry to satisfy its need for fast, strong, and cheap kind of joints suitable for wood-composites. There are many literatures supporting the idea that joints are generally the weakest part of a piece of cabinet and furniture (Eckelman 1997, Smardzewski and Prekard 2002, Eckelman 2003, Vassiliou and Barbouits 2009); they consider durability of furniture primarily on the joints rather than the composite-bodies. The present research project was conducted to find out the suitability and strength of V-nail, as a fast and easy joint in cabinet-making industry that has recently attracted the attention of craftsmen. Dovetail joint was also studied in this project to have a basis for comparison. Moreover, as the strength of joints is also significantly related to the composite panels (Eshaghi et al. 2013), two wood-composites, namely medium-density fiberboard (MDF) and particleboard (PB), were used to investigate their differences in V-nails. 


\section{MATERIALS AND METHODS}

\section{Specimen Preparation}

Laminated particleboard (PB) and medium-density fiberboard (MDF) panels $(366 \times 210 \times 16$ and $366 \times 183 \times 16 \mathrm{~mm}$ for PB and MDF, respectively) were purchased from Tehran's wood market (Iran). They were kept in conditioning chamber $\left(25 \pm 2^{\circ} \mathrm{C}, 45 \pm 3 \%\right.$ relative humidity) for two weeks before sample preparation because wood has a thermo-hygromechanical behavior and its deformation properties are significantly dependant on environmental factors such as temperature, relative humidity, and mechanical load variations (Figueroa et al. 2012). The dimensions of the composite bodies were $150 \times 50 \times 16 \mathrm{~mm}$ (the length, width, and thickness, respectively). Maximum diagonal tensile and compression loading capacities were measured in the internal and external corners of each joint (Figure 1). Once the specimens were cut and assembled, they were kept in conditioning chamber $\left(25 \pm 2^{\circ}\right.$, $45 \pm 3 \%$ relative humidity) for two weeks before tests were carried on them.

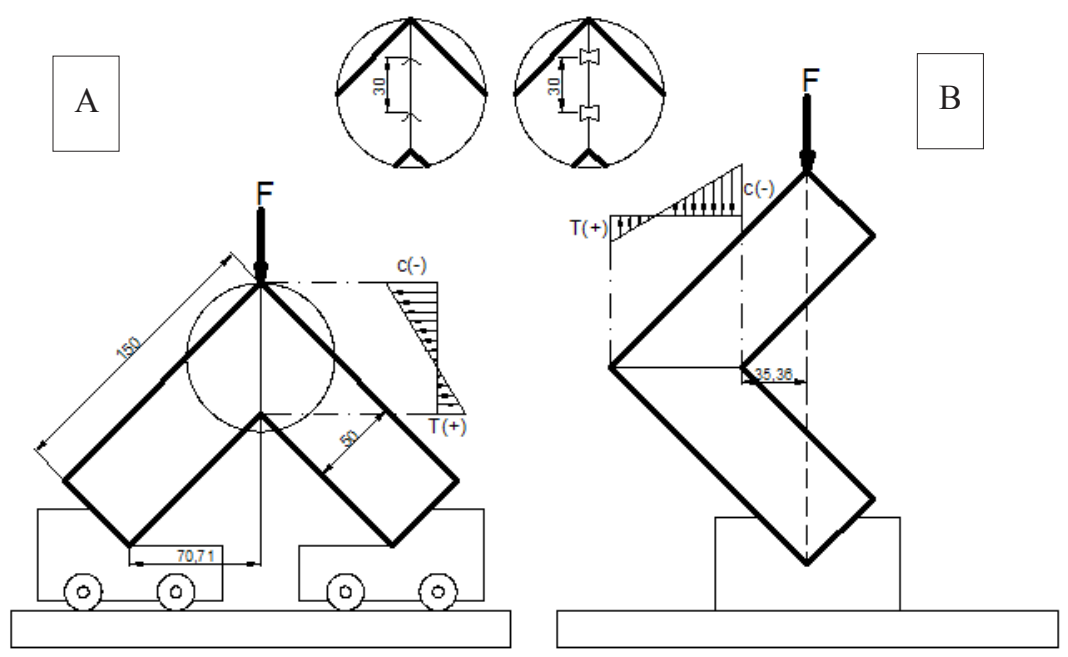

Figure 1. Schematic diagram of the loading direction to measure tensile (A) and compression (B) strength.

\section{Joint Types}

In Iran's local market, four sizes of V-nails are available, namely 7, 10, 12, and 15. Based on the popularity among craftsmen, two sizes were studied in the present research project (sizes 7 and 10) (Figure 2A). V-nails were made from hard steel. Moreover, in order to have a comparison with older joints already very common and poplar in cabinet-making industry, dovetail joint (DT joint) was also used (Figure 2B). DT joints were made from polyvinyl chloride (PVC). The height of DT joints was $14 \mathrm{~mm}$. In order to investigate the performance of each type of joint in either of the composite panels (MDF and $\mathrm{PB}$ ) used in the present study, similar sets of specimens were prepared with either of the composites.

In the meantime, polyvinyl acetate (PVA) is a common and easy-to-use type of adhesive which is very popular and common among cabinet-makers; it also provides a high performance for case construction of composite panels (Ching and Yiren 1994, Efe and Kasal 2000, Efe et al. 2002). Therefore, in order to investigate the effect of adhesive on the overall strength of the joints studied here, separate sets of joints were prepared for tensile and compression tests with PVA adhesive. The PVA used in the present study had $40 \%$ solid content based on the dry weight of the adhesive. The amounts of PVA adhesive used were 0,83 and $0,92 \mathrm{~g}$ for MDF and PB joints, respectively. For each joint type 
and size, twenty specimens were produced, ten with adhesive and ten without adhesive. Totally, 120 specimens were prepared.

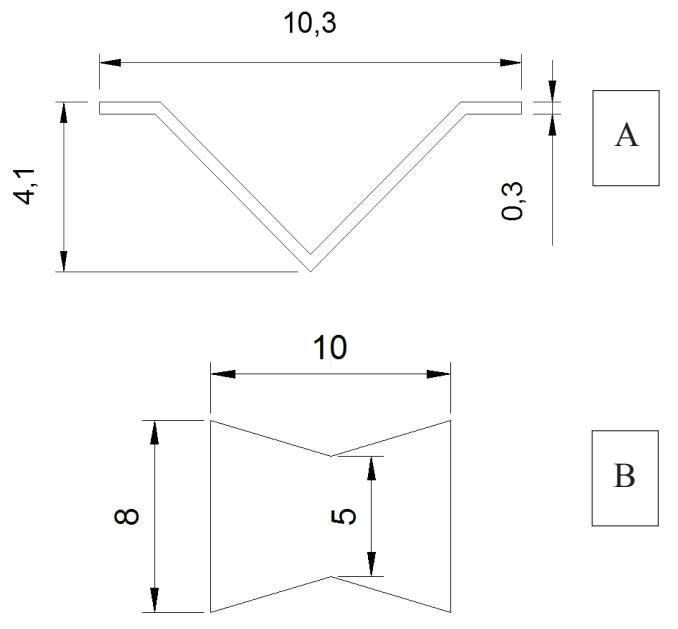

Figure 2. Schematic diagram of V-nail (A) and dovetail (B) joints.

\section{Mechanical Tests}

The tests were carried out in the mechanical laboratory of wood and wood-composites, Wood Sic. \& Tech. Dept. of Shahid Rajaee Teacher Training University (Iran). An Instron 4486 testing machine, with $5 \mathrm{kN}$ capacity, was used to carry out the tests. Specimens were positioned in the testing machine in their diagonal axis to measure tensile and compression strengths (Figure 1). The loading speed was $5 \mathrm{~mm} / \mathrm{min}$. Modulus of rupture test for the laminated particleboard was carried out in accordance with the ISIRI 9044 PB Type P2 (compatible with ASTM D1037-99) specifications. Combined stresses under diagonal tensile loading on internal and external corners were analyzed using Equations 6 and 7, respectively. In the present study, the shear strength of the adhesive applied in the joints was ignored in calculating the load-carrying capacity of joints. Based on this pre-assumption, the following Equations can be used both for joints with or without adhesive (Maleki et al. 2012, Dalvand et al. 2013). Deriving steps for Equations 6 and 7 are in Equations 1 through 5 (Dalvand et al. 2013).

$$
\begin{aligned}
& \sigma_{b}= \pm \frac{M}{S} \\
& S=\frac{1}{6} t b^{2} \\
& M=\frac{P}{2} L \\
& \sigma_{a}=\frac{-P_{a}}{2 A}=\frac{-P \cos 45}{2 b t} \\
& \sigma_{b \max }= \pm \frac{P L(6)}{2}= \pm \frac{3 P L}{t b^{2}}
\end{aligned}
$$




$$
\begin{aligned}
& \text { Internal corner }=\sigma_{b}-\sigma_{a}=\frac{3 P L}{t b^{2}}-\frac{P \cos 45}{2 b t} \\
& \text { External corner }=-\left(\sigma_{b}+\sigma_{a}\right)=-\left[\frac{3 P L}{t b^{2}}+\frac{P \cos 45}{2 b t}\right]
\end{aligned}
$$

The deriving steps to calculate stresses under compression loading on internal and external corners are indicated in Equations 8 through 17 (Dalvand et al. 2013).

$$
\begin{aligned}
& M=P(e+L)=P\left(L+\frac{b \sqrt{2}}{2}\right) \\
& e=\frac{b \sqrt{2}}{2} \\
& A=b t \\
& \sigma_{b}=\frac{M}{S} \\
& S=\frac{1}{6} t b^{2} \\
& P_{a}=P \cos 45 \\
& \sigma_{b \max }= \pm \frac{M}{S}=\frac{P\left(L+b \frac{\sqrt{2}}{2}\right)}{\frac{1}{6} t b^{2}}= \pm \frac{6 P\left(L+b \frac{\sqrt{2}}{2}\right)}{t b^{2}} \\
& \sigma_{a}=\frac{-P_{a}}{A}=\frac{-P \cos 45}{b t}
\end{aligned}
$$




$$
\begin{aligned}
& \text { Internal corner }=-\left(\sigma_{b}+\sigma_{a}\right)=-\left[\frac{6 P\left(L+\frac{b \sqrt{2}}{2}\right)}{t b^{2}}+\frac{P \cos 45}{b t}\right] \\
& \text { External corner }=\sigma_{b}-\sigma_{a}=\frac{6 P\left(L+\frac{b \sqrt{2}}{2}\right)}{t b^{2}}-\frac{P \cos 45}{b t}
\end{aligned}
$$

where, $\sigma_{a}$ is the axial stress (MPa), $\sigma_{b}$ is the bending stress (MPa), $P$ is the ultimate axial load $(\mathrm{N}), L$ is the lever arm (70,71 $\mathrm{mm}$ and $35,35 \mathrm{~mm}$ for compressive and tensile tests, respectively), $t$ is thickness of the members $(16 \mathrm{~mm}), b$ is the width of the members $(50 \mathrm{~mm})$.

\section{Statistical Analysis}

One-way analysis of variance (ANOVA) was conducted to discern significant difference at the 95\% level of confidence, using SAS/9.2 software program (2010). Grouping was then made between treatments using the Duncan's test. Hierarchical cluster analysis, including dendrogram using Ward's method with squared Euclidean distance intervals, was carried out within PAWS (SPSS software, version 18, 2010). Cluster analysis was performed to find similarities and dissimilarities between treatments based on more than one property simultaneously (Ada 2013). The scaled indicator in each cluster analysis shows how much treatments are similar or different; lower scale numbers show more similarities while higher ones show dissimilarities. Fitted-line plot was made using Minitab software, version 16.2.2 (2010).

\section{RESULTS}

Results showed that the mean diagonal tensile strengths were significantly lower in the joints without adhesive than those with PVA adhesive (Figure 3A, B, C, and C); the minimum tensile strength was found in the particleboard V-nail size 7 specimens (1 MPa). Compression strengths in the joints with adhesive were significantly higher in comparison to their counterparts; the highest compression strength was found in MDF V-nail size 10 specimens (26,5 MPa). Joints made from MDF generally showed higher strengths in comparison to joints made from particleboard.

Stress values in the external corners were all higher in comparison to those in the internal corner when tensile strength values were measured (Figure 3A, B). In the compression strength, the opposite was found; that is, stress values in the internal corners were higher (Figure 3C, D). 

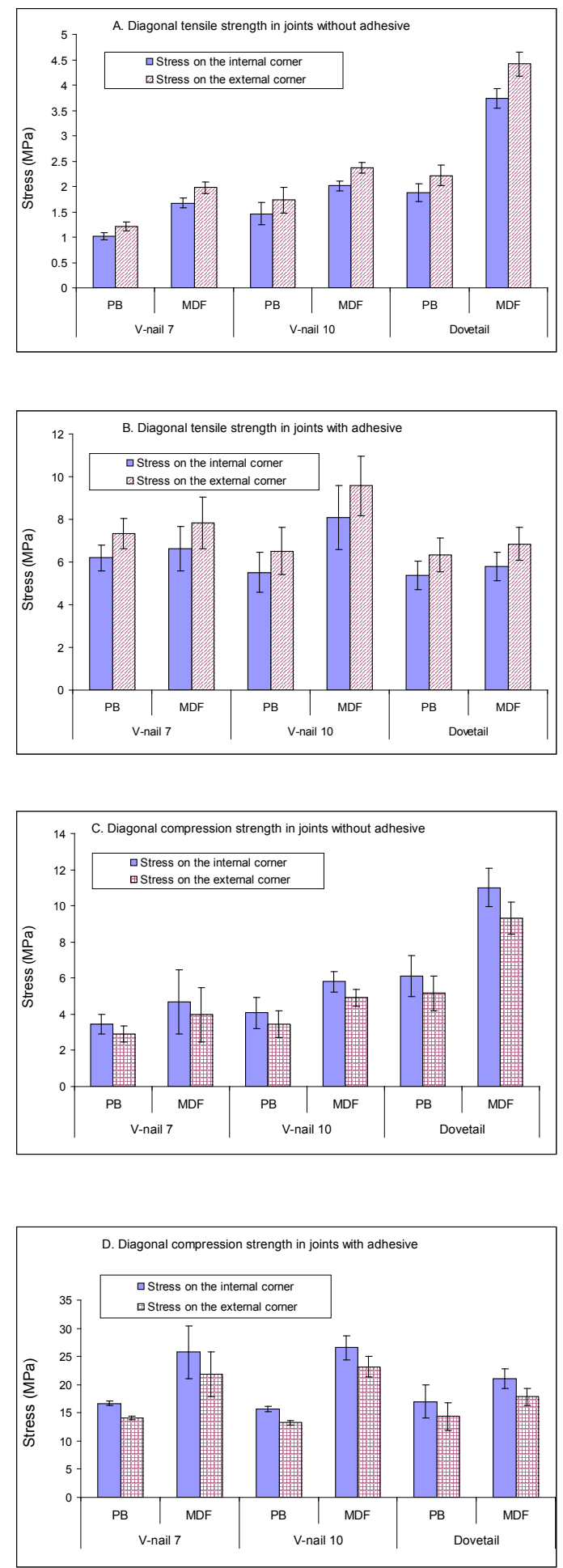

Figure 3. Diagonal load-carrying capacity values $(\mathrm{MPa})$ for tensile and compression strength in the three types of joints with and without adhesive $(\mathrm{PB}=$ particleboard; $\mathrm{MDF}=$ =medium-density fiberboard). 


\section{DISCUSSION}

In all types and combinations of joints, those made from MDF had greater load-carrying capacities (Figure 3A, B, C, D). This was due to the more integrity in MDF composites in comparison to particleboards. In fact, modulus of rupture of MDF panels was more than $47 \%$ higher in comparison to that of the particle board panels, showing higher strength and integrity in MDF (27,5 and 14,4 MPa for MDF and PB panels, respectively). Moreover, permeability in MDF was reported to be significantly lower than PB; the lower permeability in MDF indicates that the fibers are more compactly stuck together in MDF, eventually providing a more integrated and compact composite matrix.

Compression strength values showed higher values in comparison to tensile strengths in all types and combinations of joints with and without adhesive as well as composite types (Figure 3A, B, C, D). This indicated that both MDF and PB can tolerate compression stress higher than tensile stress. The stress measured in the internal and external corners showed the same trend; that is, when tensile strength was measured, the external corners illustrated greater load-carrying capacity values, having compression in the external corner (Figure 1). When compression strength was measured, the opposite was observed; the internal corner, with compression stress, demonstrated higher load-carrying capacity values. Modes of failure showed that all failures occurred in the composite members near V-nail or dovetail joints (Figure 4).
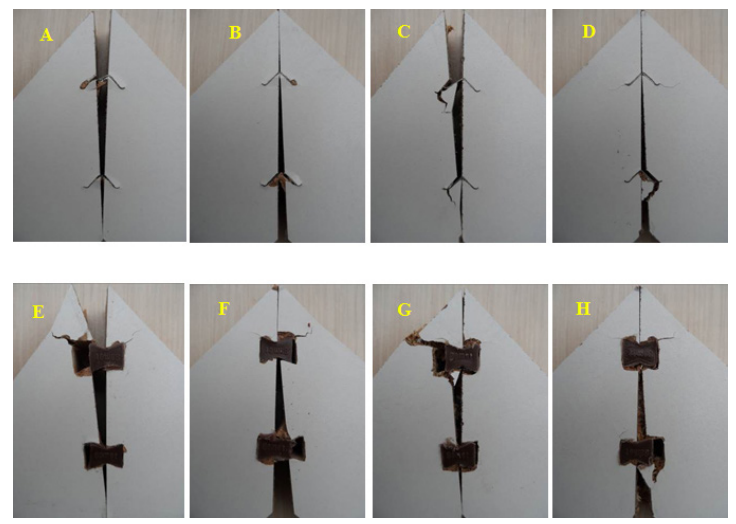

Figure 4. Some main modes of failure for V-nail and dovetail joints with members made from $\mathrm{MDF}$ and $\mathrm{PB}$ composites ( $\mathrm{PB}=$ particleboard; $\mathrm{MDF}=$ medium-density fiberboard).

(A: Compression test for V-nail in MDF with adhesive; B: Tension test for V-nail in MDF with adhesive; C: Compression test for V-nail in PB with adhesive; D: Tension test for V-nail in PB with adhesive; E: Compression test for dovetail in MDF with adhesive; F: Tension test for dovetail in MDF with adhesive; G: Compression test for dovetail in PB with adhesive; H: Tension test for dovetail in PB with adhesive)

Both tensile and compression strengths of dovetail joints without adhesive showed higher values in comparison to V-nails of either size (7 or 10) (Figure 3A, C). This indicated that dovetail joints provide craftsmen with a higher strength provided PVA adhesive is not used. The higher strengths of dovetails joints without adhesive are related to more contact area between dovetail plastic joints and composite parts. However, using adhesive in the joints, load-carrying capacity values of both tensile and compression strengths of dovetails joints were significantly lower in comparison to V-nail size 10, especially in specimens made from MDF (Figure 3C, D). In this connection, adhesive was applied in the connecting line of the two joint-members; in dovetail joints, part of the joint-members was cut out to provide space for the dovetail joint to insert (Figure 1). This significantly decreased the joint line area to be stuck with adhesive. In V-nails, however, no part of the joint line is cut out, resulting in a strong joint because of a higher bonding surface, and eventually more integrity in the joints; similar higher bonding surface was reported to increase combined stress in corner joints made from white fir (Dalvand et al. 2013). 
Load-deformation curves of both V-nail and dovetail joints without adhesives showed a steady increase upto the final bread-down point of the joints in both MDF and PB composites (Figure 5A, B). However, those curves of joints with adhesive showed formation of a mild step in the middle of the curve (Figure 5C, D); this step was as a result of the failing of the glue line between the two joint members. This indicated that adhesive carried the main load at the initial stage of loading; once the adhesive was totally failed, the dovetail and V-nail joints carried the loading. In this connection, PVA adhesive used in the present study is considered a rigid kind of glue with low flexibility; therefore, application of glues with higher flexibility may be considered for future studies to find out if the stretching of a more flexible kind of glue during the loading can improve the load-carrying capacity of V-nail and dovetail joints.
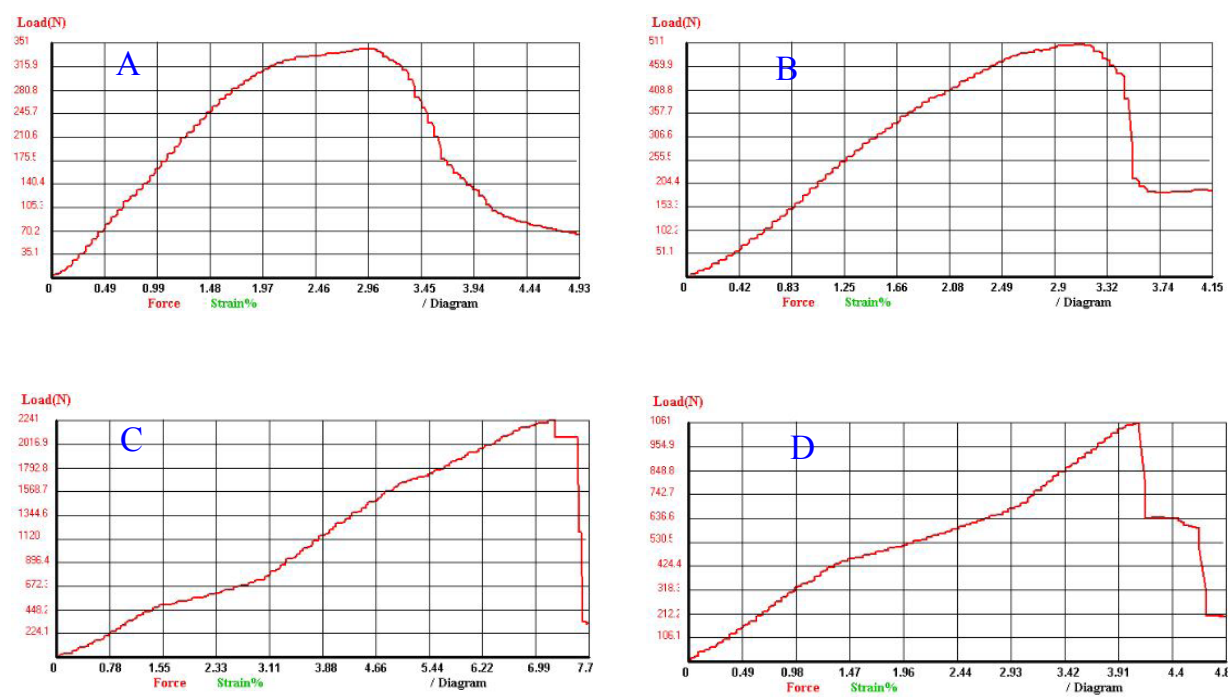

Figure 5. Load-deformation curves of V-nail and dovetail joints with members made from MDF and $\mathrm{PB}$ composites $(\mathrm{PB}=$ particleboard; $\mathrm{MDF}=$ medium-density fiberboard).

(A: Dovetail without adhesive; B: V-nail without adhesive; C: Dovetail joint with adhesive; D: V-nail with adhesive)

Fitted-line plots between tensile versus compression strengths showed high R-squares both in MDF and PB specimens (86,8 and $88 \%$ for MDF and PB, respectively) (Figure 6). This indicated that the main factors affecting the overall strength in joints would be the same for both wood-composites, regardless of the direction of force being applied to the joints. In the present research project, the main factors were the composite material as the body of joints, as well as the type and size of joints. 


\section{A. MDF}

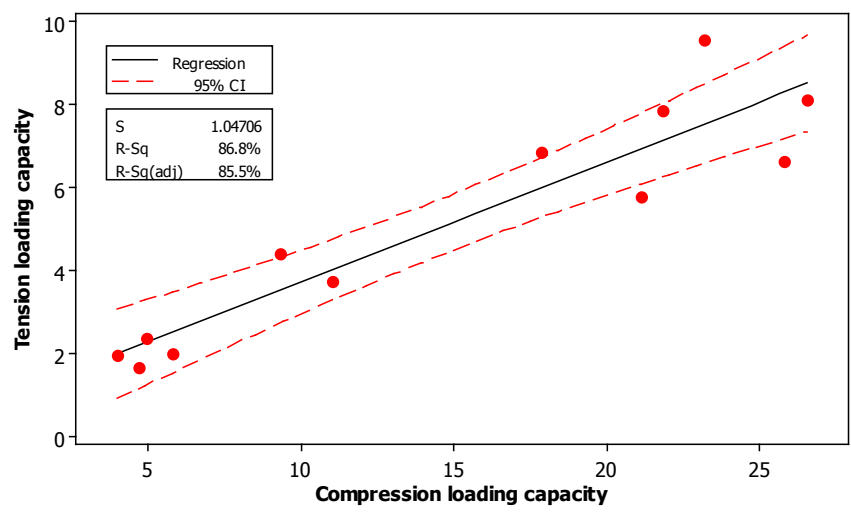

B. PB

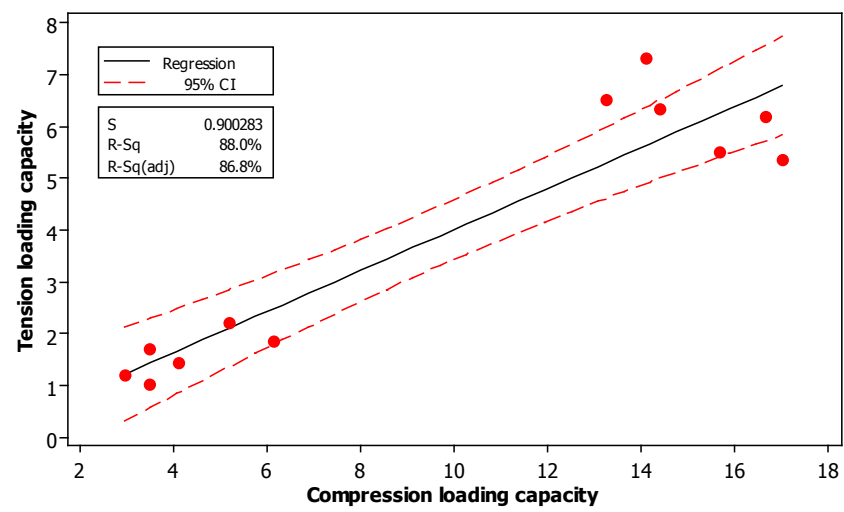

Figure 6. Fitted-line plot between tensile versus compression maximum diagonal load-carrying capacities in medium-density fiberboard (A) and particleboard (B) joint specimens with and without adhesives.

Cluster analysis of the 12 joint combinations based on the maximum tensile and compression loading capacities in the internal and external corners of the joints showed that all joints of any type with adhesive were closely clustered together (Figure 7). This indicated the significant effect of adhesive in the final loading capacity of the joints. It can therefore be concluded that application of a common adhesive such as PVA can significantly improve the load-carrying capacity of V and dovetail joints. The cluster analysis also demonstrated more similarity among joints with adhesive made from MDF or PB; this can indicate that type of wood-composite occupies the second rank for determining the ultimate load-carrying capacity of a joint. Part of the adhesive was inserted into the large cavities in PB-matrix with higher permeability, and therefore, not all applied adhesive is utilized in the process of adhering the two composite parts of joints together; in the MDF joints, however, lower permeability makes more part of the applied adhesive being present in the joint line, eventually the overall load-carrying capacity increased. Dovetail joints without adhesive made from MDF (Figure 7, No. 6) were closely clustered to its equivalent with adhesive (Figure 7, No. 12); firstly, this indicates the significant effect of the type of wood-composite panel on the overall loading capacity of the joint; and secondly, with due consideration to Figure $3 \mathrm{~A}$ and $\mathrm{C}$, it can be concluded that when craftsmen want to use joints without adhesive, dovetail joints are more recommended as their significantly higher loading capacities. 


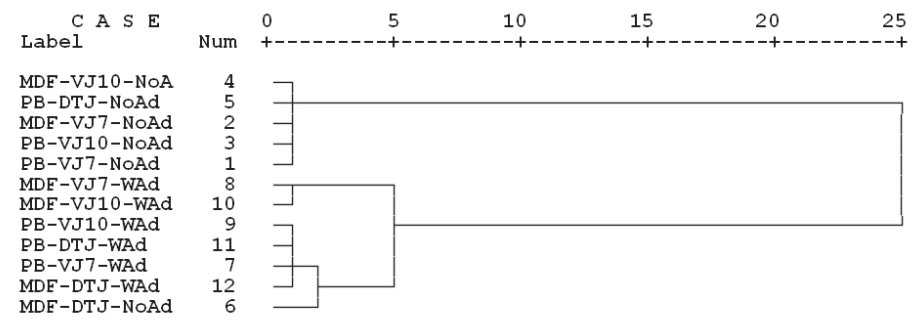

Figure 7. Cluster analysis of the 12 joints based on the maximum diagonal tensile and compression load-carrying capacities in the internal and external corners of the joints ( $\mathrm{MDF}=$ =medium-density fiberboard; $\mathrm{PB}=$ particleboard; $\mathrm{VJ} 7=\mathrm{V}$-nail size 7; $\mathrm{VJ} 10=\mathrm{V}$-nail size 10; $\mathrm{DTJ}=$ dove-tail joint; NoA=no adhesive; WAd=with adhesive).

\section{CONCLUSIONS}

Miter frame corner joints were made with V-nails sizes 7 and 10 and dovetails with and without adhesive, using medium-density fiberboard and particleboard. Tensile and compression load-carrying capacity values were measured in the internal and external corners of the joints. It was concluded that both wood-composites would demonstrate greater capacity if compression load was applied. MDF joints generally had greater load-carrying capacity values than that of PB joints; this was related to the higher compactness among wood fibers, resulting in higher integrity of the material, eventually the overall strength increased substantially. Dovetail joints demonstrated higher strengths in case no adhesive was used due to more contact area between the joint pieces and the composite bodies. When adhesive was applied, though, V-nail size 10 showed the highest strength, because part of the composite body of dovetail joint was cut out for the dovetails to be inserted, decreasing the effective contact area in the glue line of the joints. MDF joints with adhesive showed higher load-carrying capacity in comparison to $\mathrm{PB}$ joints; this was partially related to the visible cavities in PB composite matrix and its higher permeability, allowing part of the applied resin to be inserted into the cavities and being away from the process of sticking composite bodies together. Based on the results of the present research project and the cluster analysis, it was concluded that even a common adhesive such as PVA can significantly increase the load-carrying capacity of frame corner V-nail and dovetail joints in MDF and PB composites. However, in case the craftsmen prefer not to use, or cannot use, adhesives, dovetail joints provide with higher strength in comparison to V-nails sizes 7 and 10. 


\section{ACKNOWLEDGEMENTS}

The present research study was financially supported by Shahid Rajaee Teacher Training University (Tehran, Iran) for which the authors are grateful.

\section{REFERENCES}

Ada, R. 2013. Cluster analysis and adaptation study for safflower genotypes. Bulgarian Journal of Agricultural Science 19:103-109.

Ching, S.W.; Yiren, W. 1994. A study on bending moment resistance of particleboard corner joint in carcass furniture. Department of Forest Products Industry, National Chia-yi Institute of Agriculture Taiwan Forest Products Industries 13(4):600-610.

Dalvand, M.; Ebrahimi, Gh.; Rostampour-Haftkhani, A.; Maleki, S. 2013. Analysis of factors affecting diagonal tension and compression capacity of corner joints in furniture frames fabricated with dovetail key. Journal of Forestry Research 24(1):155-168.

Eckelman, C.A. 1997. Bending strength of corner joints constructed with injection-molded splines. Forest Products Journal 47(4):89-92.

Eckelman, C.A. 2003. Textbook of product engineering and strength design of furniture. Purdue University Press, West Lafayette, IN, USA.

Eshaghi, S.; Faezipour, M.; Taghiyari, H.R. 2013. Investigation on lateral resistance of joints made with drywall and sheet metal screws in bagasse particleboard and comparison with that of commercial MDF. Maderas-Cienc Tecnol 15(2):127-140.

Efe, H.; Kasal, A. 2000. Tabla tipi kavelai Kose birlestirmelerde tutkal cesidinin cekme direncine etkileri. Journal Polytech 3(4):67-72.

Efe, H.; Kasal, A.; Gurleyen, L. 2002. The Compressive strength of corner joints with dowel bonded with various types of adhesives on case construction. The Journal of The Industrial Arts Education 10(10):39-56.

Figueroa, M.; Bustos, C.; Dechent, P.; Reyes, L.; Cloutier, A.; Giuliano, M. 2012. Analysis of rheological and thermo-hygro-mechanical behaviour of stress-laminated timber bridge deck in variable environmental conditions. Maderas-Cienc Tecnol 14(3):303-319.

Maleki, S.; Derikvand, M.; Dalvand, M.; Ebrahimi, Gh. 2012. Load-carrying capacity of mitered furniture corner joints with dovetail keys under diagonal tension load. Turkish Journal of Agriculture and Forestry 36:636-643.

Smardzewski, J.; Prekard, S. 2002. Stress distribution in disconnected furniture joints. Electronic Journal of Polish Agricultural Universities, Wood Technology Series 5:1-7. 
Vassiliou, V.; Barboutis, I. 2009. Bending strength of furniture corner joints constructed with insert fittings. Annals of Warsaw University of Life Sciences - SGGW. Forestry and Wood Technology 67:268-274. 